OPEN ACCESS

Edited by:

Xu-Ming Mao,

Zhejiang University, China

Reviewed by:

Hang Wu,

Anhui University, China

Qianjin Kang,

Shanghai Jiao Tong University, China

${ }^{*}$ Correspondence:

Eung-Soo Kim

eungsoo@inha.ac.kr

Specialty section:

This article was submitted to

Synthetic Biology,

a section of the journal

Frontiers in Bioengineering and

Biotechnology

Received: 08 April 2021

Accepted: 01 June 2021

Published: 12 July 2021

Citation:

Park H-S, Nah H-J, Kang S-H,

Choi S-S and Kim E-S (2021)

Screening and Isolation of a Novel

Polyene-Producing Streptomyces

Strain Inhibiting Phytopathogenic

Fungi in the Soil Environment.

Front. Bioeng. Biotechnol. 9:692340.

doi: 10.3389/fbioe.2021.692340

\section{Screening and Isolation of a Novel Polyene-Producing Streptomyces Strain Inhibiting Phytopathogenic Fungi in the Soil Environment}

\author{
Heung-Soon Park ${ }^{1}$, Hee-Ju Nah ${ }^{1}$, Seung-Hoon Kang ${ }^{1}$, Si-Sun Choi ${ }^{1}$ and \\ Eung-Soo Kim ${ }^{1,2 *}$
}

\begin{abstract}
${ }^{1}$ Department of Biological Sciences and Bioengineering, Inha University, Incheon, South Korea, ${ }^{2}$ Department of Biological Engineering, Inha University, Incheon, South Korea
\end{abstract}

Microbial-based eco-friendly biological substances are needed to protect crops from phytopathogenic fungi and replace toxic chemical fungicides that cause serious environmental issues. This study screened for soil antifungal Streptomyces strains, which produce rich, diverse, and valuable bioactive metabolites in the soil environment. Bioassay-based antifungal screening of approximately 2,400 Streptomyces strains led to the isolation of 149 strains as tentative antifungal producers. One Streptomyces strain showing the most potent antifungal activities against Candida albicans and Fusarium oxysporum was identified as a putative anti-phytopathogenic soil isolate that is highly homologous to Streptomyces rubrisoli (named S. rubrisoli Inha 501). An in vitro antifungal assay, pot-test, and field-test against various phytopathogenic fungi confirmed that S. rubrisoli Inha 501 is a potential novel phytopathogenic fungicide producer to protect various crops in the soil environment. Whole-genome sequencing of S. rubrisoli Inha 501 and an anti-SMASH genome mining approach revealed an approximately 150-kb polyene biosynthetic gene cluster (BGC) in the chromosome. The target compound isolation and its BGC analysis confirmed that the giant linear polyene compound exhibiting the anti-phytopathogenic activity in S. rubrisoli Inha 501 was highly homologous to the previously reported compound, neotetrafibricin A. These results suggest that a bioassay-based screening of a novel antifungal Streptomyces strain followed by its genome mining for target compound BGC characterization would be an efficient approach to isolating a novel candidate phytopathogenic fungicide that can protect crops in the soil environment.

Keywords: Streptomyces, phytopathogenic fungicide, polyene, BGC, genome mining, biosynthetic gene cluster

\section{INTRODUCTION}

For a long time in traditional agriculture, chemical fungicides have been used to control phytopathogenic fungi, such as Fusarium, Botrytis, and Colletotrichum, which cause severe damage in crop production (Dean et al., 2012; Parnell et al., 2016). On the other hand, the use of chemical fungicides has been restricted because of human toxicity and ecosystem 
destruction (Ab Rahman et al., 2018). Alternative methods are being pursued to protect crops from phytopathogenic fungi using soil-rich microorganisms, such as Pseudomonas, Bacillus, and Streptomyces species, to minimize these toxic compounds in the soil environment. Several commercial products containing these microbial strains are potential biocontrol agents against various phytopathogenic fungi (Parnell et al., 2016; Ab Rahman et al., 2018; Abbasi et al., 2019). Among them is a genus called Streptomyces, a high G + C Gram-positive bacteria, which are ubiquitous in the soil environment. Streptomyces produce various useful bioactive secondary metabolites, such as antibiotics, antiviral, anticancer, anti-inflammatory, antiparasitic, and antioxidant compounds (Manivasagan et al., 2014; JakubiecKrzesniak et al., 2018; Bu et al., 2019). In recent years, many Streptomyces species present in plant roots have also shown beneficial effects on crops by controlling phytopathogenic fungi or the secretion of plant growth hormones and increasing the possibility of agricultural applications (Shi et al., 2018; Kim et al., 2019).

Polyene compounds typically comprise a polyketide core macrolactone ring with $20-40$ carbon atoms, including 3-8 conjugated double bonds. The most characterized antifungal polyenes used primarily to treat severe fungal infections are polyene macrolides, such as the tetraene-containing nystatin A1 and heptaene-containing amphotericin B (Caffrey et al., 2016; Zhang et al., 2017). The primary antifungal mechanism of polyene antimicrobials is dependent on the interactions between the antibiotic molecules and ergosterol that appear to occur through the polyene region of the macrolactone core. In addition to these typical macrocyclic polyene compounds, there are also linear aminopolyol polyene compounds containing an amino- or guanidino-moiety, such as linearmycin, ECO02301, mediomycin, and neotetrafibricin (Figure 1, Caffrey et al., 2016; Zhang et al., 2017). The polyene core is biosynthesized by a multi-modular giant enzyme complex called polyketide synthase (PKS), and the following processes are programmed by each domain included in PKS. In general, the acyltransferase (AT) domain selects and loads an extender unit (acyl-CoA) to the acyl-career protein (ACP) domain and the keto-synthase (KS) domain then catalyzes a decarboxylative condensation. Optionally, the keto-reductase (KR), dehydratase $(\mathrm{DH})$, and enoylreductase (ER) domains reduce the $\beta$-keto group to a $\beta$-hydroxyl group, an $\alpha, \beta$-double bond, and a saturated bond, respectively. Finally, the thioestrease (TE) domain cleaves the polyketide chain from PKS. The chain is modified by post-PKS modification enzymes, including P450 hydroxylases and glycosyltransferases (Caffrey et al., 2016; Zhang et al., 2017). The major antifungal mechanism of these polyenes is considered the formation of ion channels via fungal ergosterol binding that mediates the leakage of cellular $\mathrm{K}^{+}$and $\mathrm{Mg}^{2+}$, which leads to the death of fungal cells (Neumann et al., 2016).

The traditional activity-based screening strategy is still the most widely practiced approach for selecting useful and diverse bioactive metabolites produced by soil Streptomyces species. Recently, alternative state-of-the-art technologies, including microbial genome mining, BGC cloning, heterologous expression, and pathway refactoring, have been pursued to complement the weaknesses of the traditional approaches, such as re-isolation and low expression issues under typical laboratory culture conditions (Lee et al., 2020). In particular, the genome mining strategy is an approach that analyzes and utilizes functional microbial genomes through bioinformatic analyses, enabling the establishment of effective strategies for predicting the pathways of various bioactive metabolite biosynthetic gene clusters (BGCs) present in the bacteria (Zerikly and Challis, 2009; Lee et al., 2020). Although a bioinformatics-based prediction using a genome mining approach still needs to be supplemented through extensive laboratory work, novel bioactive compounds can be synthesized from bacteria to various derivatives in the desired location, or natural compounds can be synthesized using sophisticated and rational reprogramming (Ziemert et al., 2016; Awakawa et al., 2018).

In this study, the bioassay-based antifungal screening of approximately 2,400 Streptomyces strains led to the isolation of 149 strains as tentative antifungal producers. One of these Streptomyces strains, showing the most potent antifungal activities against Candida albicans and Fusarium oxysporum, was identified as a putative polyene-producing soil isolate, highly homologous to Streptomyces rubrisoli (named S. rubrisoli Inha 501). An in vitro antifungal assay, pot-test, and field-test against various phytopathogenic fungi confirmed that $S$. rubrisoli Inha 501 could be a good candidate for a novel phytopathogenic fungicide to protect various crops in the soil environment.

\section{MATERIALS AND METHODS}

\section{Strains and Growth Conditions}

S. rubrisoli Inha501 was distributed from Industrial Biomaterial Research Center, Korea Research Institute of Bioscience and Biotechnology (KRIBB), South Korea. The strain was grown routinely in ISP2 agar (malt extract $10 \mathrm{~g}$, yeast extract $4 \mathrm{~g}$, glucose $4 \mathrm{~g}$, and agar $20 \mathrm{~g}$ per liter) at $30^{\circ} \mathrm{C}$ for the sporulation and seed culture. The R5 medium (sucrose $51.5 \mathrm{~g}$ yeast extract $2.5 \mathrm{~g}$, peptone $5 \mathrm{~g}$, malt extract $3 \mathrm{~g}$, glucose $10 \mathrm{~g}$, sucrose $340 \mathrm{~g}$, and $10 \mathrm{~N}$ $\mathrm{NaOH} 0.7 \mathrm{ml}$ per liter) was used to produce the I-NTF (Inhaneotetrafibricin A). All Escherichia coli strains were incubated at $37^{\circ} \mathrm{C}$ in Luria-Bertani medium supplemented with the appropriate antibiotics where needed. Candida albicans ATCC 14053, Aspergillus niger ATCC 9642, Fusarium oxysporum f. sp. lactucae KACC (Korean Agricultural Culture Collection) 42795, Fusarium oxysporum $\mathrm{f}$. sp. gladioli KACC 40051, Fusarium solani KACC 44891, Fusarium graminearum KACC 47495, Fusarium verticillioides KCTC (Korean Collection for Type Cultures) 6065, Fusarium semitectum KCTC 16672, Botrytis cinerea KACC 40574, Colletotrichum gloeosporioides KACC 40003, Curvularia lunata KACC 40861, and Alternaria alternata KACC 40019 were grown on PDA medium (potato starch $4 \mathrm{~g}$, glucose $2 \mathrm{~g}$, agar $15 \mathrm{~g}$ per liter) at $28^{\circ} \mathrm{C}$ for $2-7$ days.

\section{Antifungal Pot-Test and Field-Test}

The fermentation TSB broth of $S$. rubrisoli Inha 501 was tested for in vivo antifungal activity against Fusarium wilt. In the case 
A

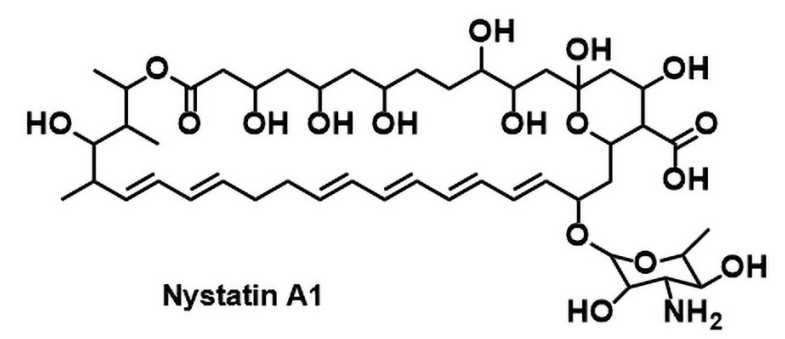<smiles>CC=CC=CC=CC=CC=CC=CC=CC(CC1OC(O)(CC(O)CC(O)C(O)CCC(O)CC(O)CC(=O)OC(C)C(O)C(C)C)CC(O)C1C(=O)O)OC1OC(C)C(N)C(O)C(C)O1</smiles>

Amphotericin B

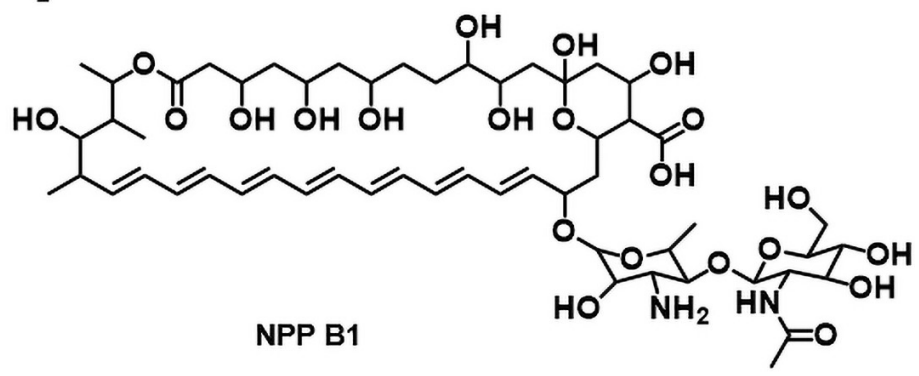

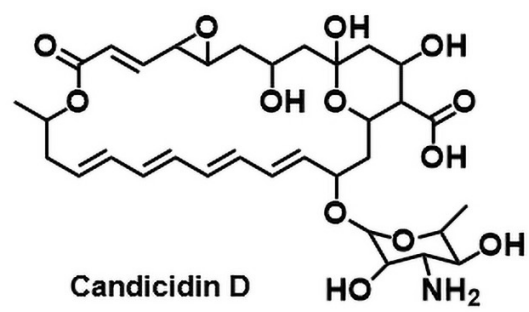

NPP B1

B
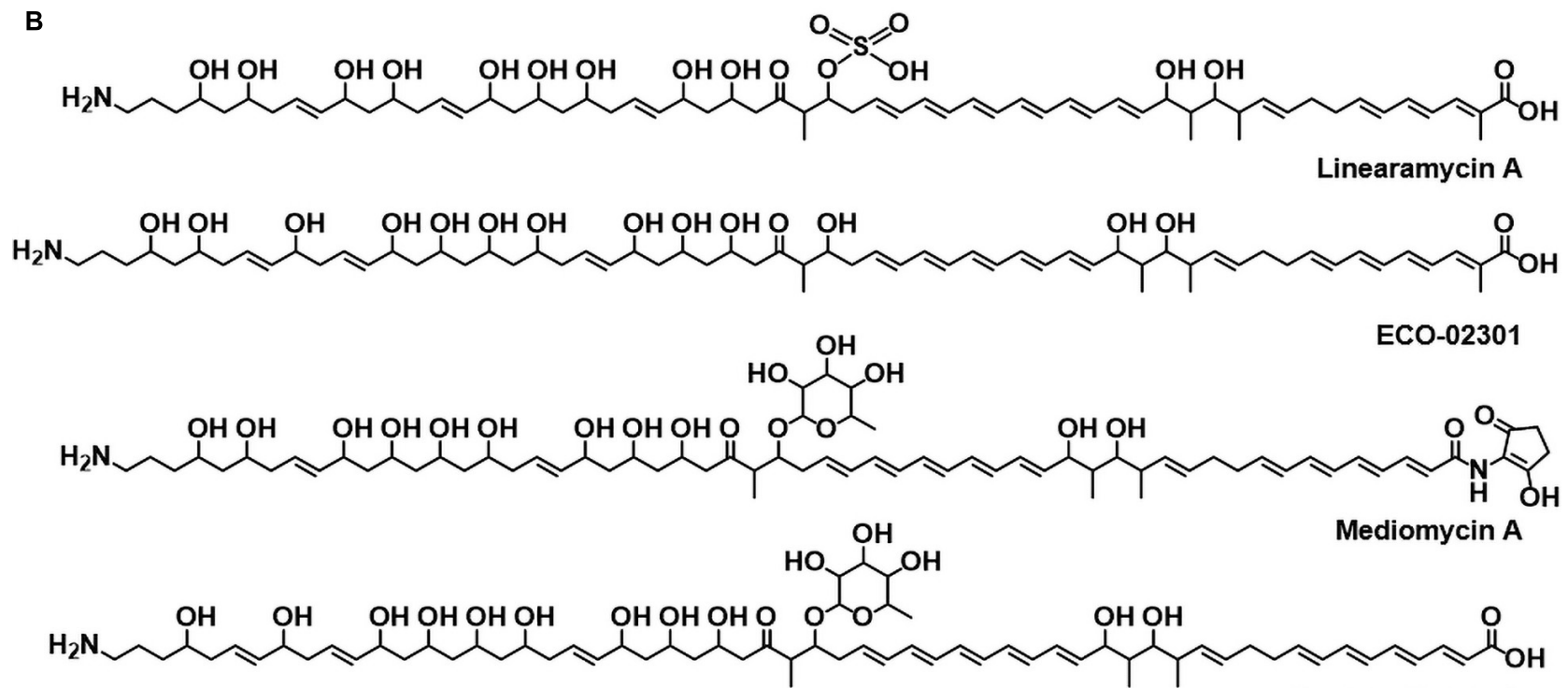<smiles>CCCCCCCCC(O)CC(C)O</smiles>

FIGURE 1 | (A) Circular polyenes; nystatin A1, amphotericin B, candicidin D, and natamycin. (B) Linear polyenes; linearmycin A, ECO-02301, mediomycin A, neotetrafibricin A.

of the pot-test, eight of each red pepper, strawberry, and tomato seedlings were prepared, respectively. The prepared seedlings were grown for 2 weeks in vinyl pots (diameter $10 \mathrm{~cm}$ ) in a growth room at $22 \pm 1{ }^{\circ} \mathrm{C}$. Four seedlings of tree crops were treated with $100 \mathrm{ml}$ of $S$. rubrisoli Inha501 culture medium $\left(10^{6} \mathrm{CFU} / \mathrm{ml}\right)$ along with a negative control treated only with $100 \mathrm{ml}$ of TSB. After $24 \mathrm{~h}$, the potted seedlings were wounded on the stem and then treated with F. oxysporum KACC 40051. After 2 weeks, the appearance of the seedlings was examined to measure the degree of Fusarium wilt infection (0: disease-free 100: crop failure). The pot-to-pot significance difference test was performed at the $95 \%$ level by the DMRT method (Kramer, 1957). In the case of field tests, tests were conducted in accordance with "the pesticide registration test standards and methods" in Korea (Rural Development Administration, 2008). In the evaluation, the minimum incidence rate in the untreated seedlings was more than $10 \%$, and the effectiveness was assessed by comparing the morbidity rate with the untreated seedlings 10 days after the final S. rubrisoli Inha501 treatment. As for the placement of the test seedlings, the test was carried out in three repetitions of the randomized complete block design and the complete randomized design (Zerbe, 1979; Scott and Milliken, 1993). Two red pepper and tomato greenhouses each infected with Fusarium wilt were prepared. The prepared seedlings were grown in soil $\left(10 \mathrm{~m}^{2}\right)$ in a growth room at $29 \pm 3^{\circ} \mathrm{C}$ for 4 weeks. The test seedlings were treated with $10 \mathrm{~L}$ of $S$. rubrisoli Inha501 culture medium 
$\left(2 \times 10^{3} \mathrm{CFU} / \mathrm{ml}\right)$ and the untreated seedlings were treated with $10 \mathrm{~L}$ of TSB. The pot-to-pot significance difference test was performed at the $95 \%$ level by the DMRT method (Kramer, 1957). The more detailed results of antifungal pot-test and field-test are provided as Supplementary Figure 2.

\section{Genome Sequencing and Assembly of S. rubrisoli Inha 501}

The S. rubrisoli Inha501 genome was sequenced at Macrogen (Korea) using both the PacBio RSII (Pacific Biosciences, United States) and Illumina HiSeq (Illumina, United States) platforms. The library preparation for Illumina and PacBio sequencing was performed using the TruSeq DNA sample prep kit for Illumina (NE, United States) and the PacBio DNA Template Prep Kit 1.0 (Pacific Biosciences, United States), respectively. The library insert sizes were $350 \mathrm{bp}$ for Illumina sequencing and $20 \mathrm{~kb}$ for PacBio RS SMRT sequencing. The de novo assembly of sequenced fragments was performed using Canu (v1.7) software. A high-quality sequence was obtained by performing an error correction of the assembled contig using Pilon (v1.21) software. The annotation was performed using Prokka (v1.12b) software.

\section{Production and Purification of Polyene Compound}

The S. rubrisoli Inha501 was inoculated in $200 \mathrm{ml}$ of TSB medium at $30^{\circ} \mathrm{C}$ and $220 \mathrm{rpm}$ for $48 \mathrm{~h}$. The pre-cultures were added to $2 \mathrm{~L}$ of $\mathrm{R} 5$ medium in a $5 \mathrm{~L}$ bioreactor for batch fermentation. After $120 \mathrm{~h}$ of cultivation, the culture broth was extracted in $2 \mathrm{~L}$ of $n$-butanol. The extract was concentrated using a vacuum evaporator. The concentrated extract was dissolved in methanol and loaded onto a column packed with a C18 reversed-phase silica gel (Daiso, Japan) and eluted with methanol-water (30:70, $\mathrm{v} / \mathrm{v})$ to remove any residual sugar from the production media. The extracts with the sugar removed were purified using a fraction collector (Interchim, France) on a gradient comprised of solvents A (water) and B (methanol): $30 \% \mathrm{~B}(\mathrm{v} / \mathrm{v})(0-10 \mathrm{~min}$ ) and $100 \% \mathrm{~B}(\mathrm{v} / \mathrm{v})(100 \mathrm{~min})$ at a flow rate of $20 \mathrm{ml} / \mathrm{min}$. The fractions that contained I-NTF with $>90 \%$ purity were detected at $332 \mathrm{~nm}$ and analyzed by high-performance liquid chromatography (HPLC). The column was equilibrated with $60 \%$ solvent A (0.05 M ammonium acetate, $\mathrm{pH} 6.5$ ) and $40 \%$ solvent $\mathrm{B}$ (acetonitrile). The flow rate was set to $0.5 \mathrm{ml} / \mathrm{min}$ under the following conditions: $0-30 \mathrm{~min}, 40 \% \mathrm{~B}$.

\section{LC-MS/MS Analysis}

The polyene compound showing $>90 \%$ purity was analyzed using A Triple TOF 5,600 + (AB Sciex, United States) coupled with Ultimate3000 (Thermo Scientific, United States). Mass spectrometry was conducted in both positive and negative ion modes over a mass range from $\mathrm{m} / z 50$ to 2,000 using an electrospray ionization source. The settings were nitrogen gas for nebulization at $50 \mathrm{psi}$, heater gas pressure at $50 \mathrm{psi}$, curtain gas at $25 \mathrm{psi}$, temperature of $500^{\circ} \mathrm{C}$, and an ion spray voltage at $5,500 \mathrm{~V}$ in positive ion mode and $-4,500 \mathrm{~V}$ in negative ion mode. The optimized declustering potential (DP) and collision energy (CE) were set to 60 and $10 \mathrm{eV}$ in positive ion mode, and -60 and $-10 \mathrm{eV}$ in negative ion mode, respectively. A sweeping collision energy setting at $35 /-35 \mathrm{eV} \pm 15 \mathrm{eV}$ was applied for collision-induced dissociation (CID). For the chromatographic conditions, solutions A $(0.1 \%$ formic acid in distilled water $)$ and $\mathrm{B}(0.1 \%$ formic acid in acetonitrile) were used for elution and loaded onto a Phenomenex Kinetex $1.7 \mu \mathrm{C} 18(2.1 \mathrm{~mm} \times$ $150 \mathrm{~mm}, 1.7 \mu \mathrm{m})$. The flow rate was set to $0.4 \mathrm{ml} / \mathrm{min}$ under the following conditions: $0-1 \mathrm{~min}, 90 \% \mathrm{~A} ; 1-5 \mathrm{~min}, 90-50 \% \mathrm{~A}$; 5$18 \mathrm{~min}, 50-0 \% \mathrm{~A} ; 18-25 \mathrm{~min}, 0 \% \mathrm{~A} ; 25-27 \mathrm{~min}, 0-90 \% \mathrm{~A}$; and 27-30 $\mathrm{min}, 90 \% \mathrm{~A}$.

\section{Inactivation of I-NTF PKS I Gene}

An I-NTF PKS I gene inactivation cassette, including homologous region of $I-N T F$ PKS $I$ gene, was constructed by PCR amplification using the following primer pairs: DELL_F (5'-GGCCA GTGCCAAGCTTCCTCGACGGGATCCGCGT-3') and DELL_R (5'- ACATGATTACGAA TTGATCGCACTACC ACGAACCA $-3^{\prime}$ ) (Supplementary Figure 5). The amplified fragments were ligated into pKC1132 digested with HindIII using an In-Fusion Cloning kit (Takara Bio, Japan). The I-NTF $P K S I$ gene inactivation cassette was then introduced into E. coli ET12567/pUZ8007 and conjugated directly with S. rubrisoli Inha501 by homologous recombination. The desired mutant was selected on apramycin-included ISP2 agar medium, and its genotypes were verified by PCR.

\section{In vitro Assays for Biological Activities}

The Clinical and Laboratory Standards Institute document M27A3 was adapted to the in vitro antifungal assay (Clinical and Laboratory Standards Institute, 2008). After fungus was cultured in $\mathrm{PDB}$ medium at $30^{\circ} \mathrm{C}$ for $1 \sim 7$ days, the cultured solution was diluted with $\mathrm{PDB}$ medium until the $\mathrm{OD}$ value reached 0.3 at $530 \mathrm{~nm}$. A working suspension was prepared by a 1:2,000 dilution with RPMI-1640 broth media (with glutamine and phenol red, without bicarbonate, Sigma-Aldrich, United States), which resulted in $5.0 \times 10^{2}$ to $2.5 \times 10^{3}$ cells per $\mu$ l. Ten microliter of the DMSO containing polyene antibiotics at various concentrations $(25-1,600 \mu \mathrm{g} / \mathrm{ml})$ were added to the working suspension of $990 \mu \mathrm{l}$ and the mixtures were then incubated at $30^{\circ} \mathrm{C}$ without shaking for $2 \sim 7$ days. The colorimetric change in the mixture from red to yellow indicated the growth of the fungus. The minimum inhibitory concentration (MIC) was determined by measuring the minimum concentration that changed the color to yellow. The experiment was performed in duplicate.

\section{RESULTS}

\section{Screening and Isolation of an Antifungal Soli Streptomyces Species}

A total of 2,419 Streptomyces culture extracts provided from the actinomycetes cell collection center in the Industrial Biomaterial Research Center, Korea Research Institute of Bioscience and Biotechnology (KRIBB), Korea was screened to isolate the strains 
showing antifungal activities against $C$. albicans (a fungal strain for the confirmation of antifungal activity) and $F$. oxysporum (a phytopathogenic fungal strain) (Figure 2 and Supplementary Figure 1). 149 antifungal culture extracts exhibiting antifungal activity were first selected, followed by the additional selection of 51 antifungal strains showing a typical polyene spectrum assayed by 2-dimensional HPLC analysis (Figure 2, Guo et al., 2000). Among the 51 strains tested, one Streptomyces sp. isolated from organic green tea fields in Jeolla Province in Korea $\left(34^{\circ} 51^{\prime} 31.7^{\prime \prime} \mathrm{N} 127^{\circ} 08^{\prime} 48.1^{\prime \prime} \mathrm{E}\right)$ was selected as a final candidate to control phytopathogenic fungi, showing the strongest antifungal activities against $C$. albicans and F. oxysporum. 16s rRNA sequence-based phylogenetic analysis showed that the strain isolated above exhibited $98.9 \%$ similarity of $S$. rubrisoli FXJ1.725 (KC137300) and was called S. rubrisoli Inha501 (Figure 3A).
Laboratory-scale antifungal bioassays were performed to assess the potential applications for the development of phytopathogenic fungicide using the isolated S. rubrisoli Inha501 against various phytopathogenic fungi, F. oxysporum, $F$. solani, F. graminearum, F. verticilliodes, F. semitectum, Aspergillus niger, Botrytis cinerea, Colletotrichum gloeosporioides, Curvularia lunata, and Alternaria alternata. As shown in Figure 3B, S. rubrisoli Inha501 showed a strong and broad spectrum of phytopathogenic antifungal activities against most phytopathogenic fungi tested.

The controllability of phytopathogenic fungi in several crops was tested by performing pot-scale tests with the $S$. rubrisoli Inha501 culture in the plants infected with $F$. oxysporum (KACC 40051). As shown in Table 1A, the fungal pathogen control rate for each red pepper, strawberry, and tomato was
A

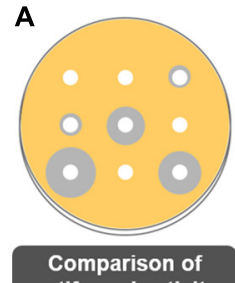

antifungal activity

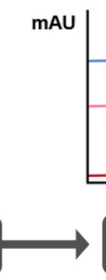

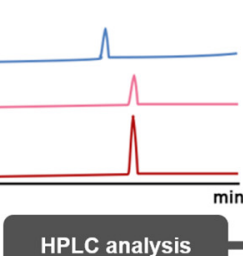

HPLC analysis

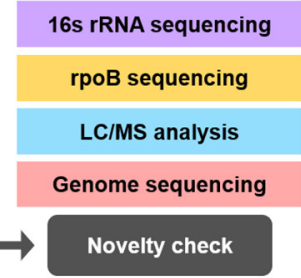

B

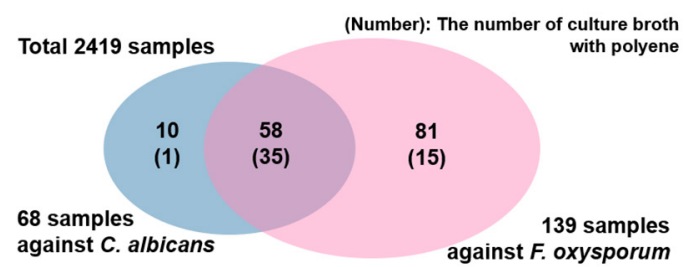

FIGURE 2 | (A) Antifungal bioassay-based screening scheme. (B) Venn diagram showing the number of strains showing antifungal activities against C. albicans and F. oxysporum.

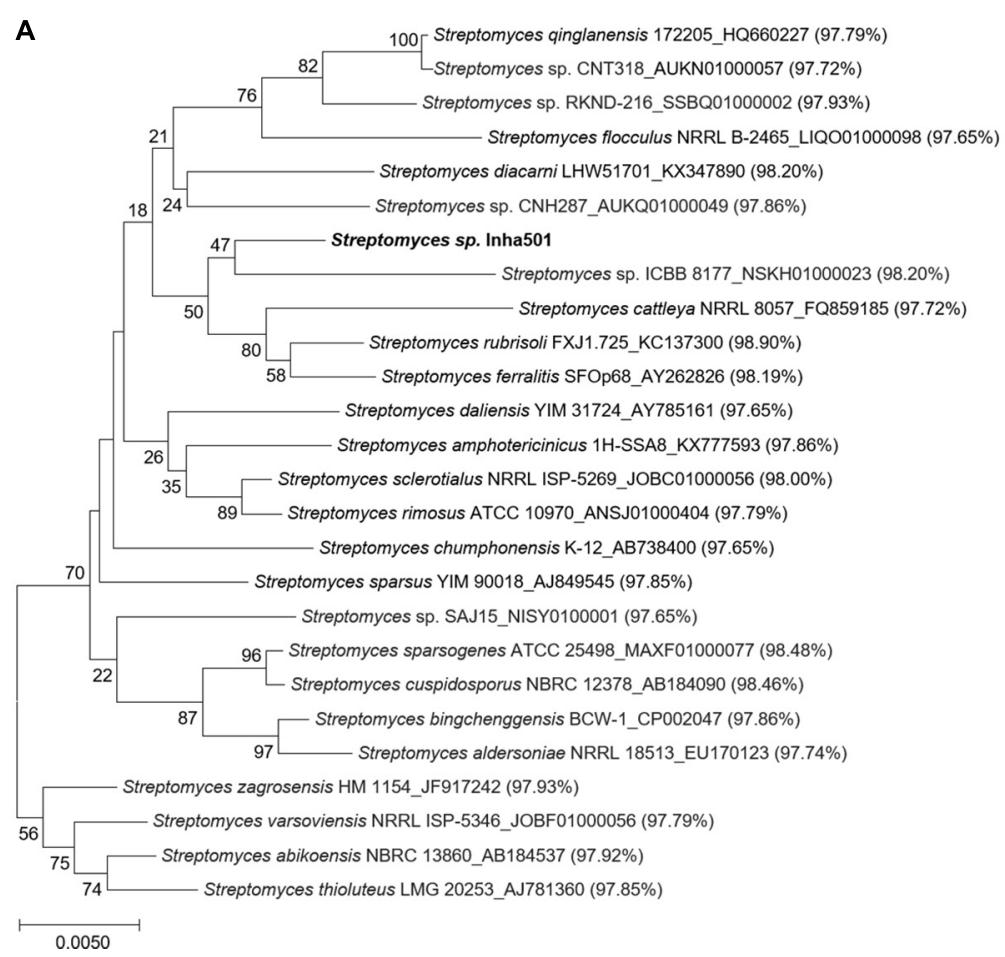

B
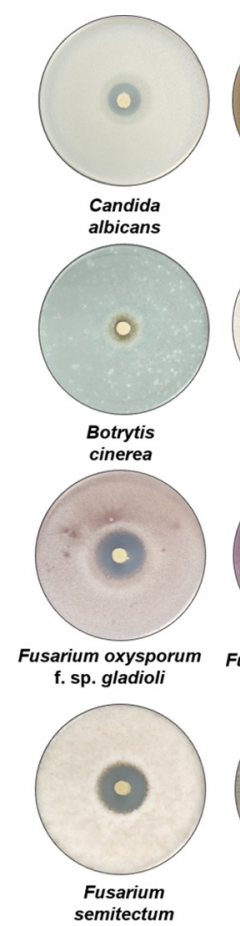

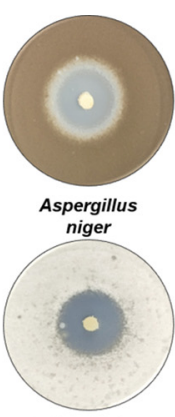

Colletotrichum gloeosporioides
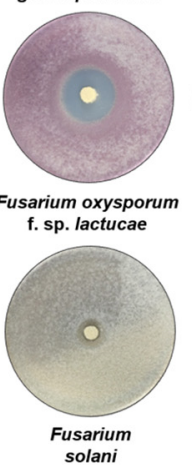

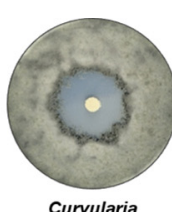

Curvularia

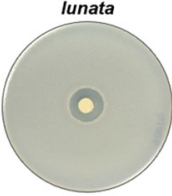

Alternaria alternata

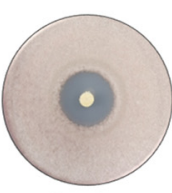

Fusarium

verticillioides

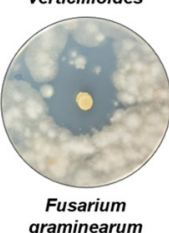

FIGURE 3 | (A) Phylogenetic tree of S. rubrisoli Inha501 based on the 16s rRNA sequence; the evolutionary history was inferred using the Neighbor-Joining method. Evolutionary analyses were conducted in MEGA7 (Kumar et al., 2016). (B) Antifungal assays of S. rubrisoli Inha501 against various phytopathogenic fungi. 
TABLE 1 | In vivo antifungal activity of S. rubrisoli Inha501 against Fusarium wilt.

\begin{tabular}{|c|c|c|c|c|c|c|c|c|c|}
\hline \multirow[t]{2}{*}{ Pot test } & \multicolumn{3}{|c|}{ Tomato $^{a}$} & \multicolumn{3}{|c|}{ Strawberry $^{b}$} & \multicolumn{3}{|c|}{ Red pepper ${ }^{\mathrm{C}}$} \\
\hline & $\begin{array}{c}\text { Disease } \\
\text { incidence }(\%)\end{array}$ & DMRT & $\begin{array}{l}\text { Control } \\
\text { rate }(\%)\end{array}$ & $\begin{array}{c}\text { Disease } \\
\text { incidence }(\%)\end{array}$ & DMRT & $\begin{array}{l}\text { Control } \\
\text { rate }(\%)\end{array}$ & $\begin{array}{c}\text { Disease } \\
\text { Incidence (\%) }\end{array}$ & DMRT & $\begin{array}{l}\text { Control } \\
\text { rate }(\%)\end{array}$ \\
\hline Inha501 & 35.0 & bc & 63.2 & 15.0 & $b$ & 69.2 & 23.1 & bc & 70.6 \\
\hline No-treatment & 95.0 & $\mathrm{a}$ & - & 48.8 & $\mathrm{a}$ & - & 78.8 & $\mathrm{a}$ & - \\
\hline
\end{tabular}

Coefficient of variation - ${ }^{a} 13.0 \%,{ }^{b} 21.2 \%,{ }^{c} 19.3 \%$

(B) Field-test against Fusarium wilt

\begin{tabular}{|c|c|c|c|c|c|c|c|}
\hline \multirow[t]{2}{*}{ Field test $\mathrm{I}^{\mathrm{a}}$} & \multicolumn{3}{|c|}{ Tomato } & \multirow[t]{2}{*}{ Field test $I^{b}$} & \multicolumn{3}{|c|}{ Tomato } \\
\hline & Disease incidence (\%) & DMRT & Control rate (\%) & & Disease incidence (\%) & DMRT & Control rate (\%) \\
\hline Inha501 & 5.3 & $\mathrm{~b}$ & 65.0 & Inha501 & 6.3 & $\mathrm{~b}$ & 51.5 \\
\hline No-treatment & 15.4 & a & - & No-treatment & 13.1 & a & - \\
\hline \multirow[t]{2}{*}{ Field test IIIC } & \multicolumn{3}{|c|}{ Red pepper } & Field test IV ${ }^{d}$ & \multicolumn{3}{|c|}{ Red pepper } \\
\hline & Disease incidence (\%) & DMRT & Control rate (\%) & & Disease incidence (\%) & DMRT & Control rate $(\%)$ \\
\hline Inha501 & 5.1 & $\mathrm{~b}$ & 57.5 & Inha501 & 10.5 & $\mathrm{~b}$ & 55.1 \\
\hline No-treatment & 12.0 & $\mathrm{a}$ & - & No-treatment & 23.14 & $\mathrm{a}$ & - \\
\hline
\end{tabular}

Coefficient of variation $-{ }^{a} 15.5 \%,{ }^{b} 10.7 \%,{ }^{c} 17.9 \%,{ }^{d} 29.4 \%$.

observed at 70.6, 69.2, and 63.2\%, respectively. Moreover, the fungal pathogen control rate in the field-scale test was 56.3 and $58.2 \%$ for the naturally infected red pepper and tomato, respectively, implying a positive outlook for its registration as a biocontrol microbial agent (Table $\mathbf{1 B}$ and Supplementary Figure 2).

\section{Whole-Genome Sequencing and Characterization of Biosynthetic Gene Clusters}

Whole-genome sequencing was performed to identify the biosynthetic gene cluster (BGC) present in the S. rubrisoli Inha501 chromosome, which is responsible for producing the target antifungal compound. The complete genome size was $8,249,972 \mathrm{bp}$, and the $\mathrm{G}+\mathrm{C}$ content was $70.19 \%$ (Figure 4A and Supplementary Table 1). The whole genome sequence was deposited in Strategic Initiative for Microbiomes in Agriculture and Food ${ }^{1}$ with an accession number igem-0000408. The S. rubrisoli Inha501 genome contained 2 plasmids $(85,489,32,253$ bp), 7,318 genes, 75 tRNAs, and 18 rRNAs (Supplementary Table 1). The encoding gene sequences were aligned with the eggnog databases to predict the putative gene functions (Huerta-Cepas et al., 2019). Among them, 6,881 genes were successfully annotated with Eggnog, accounting for $94 \%$ of all genes (Figure 4B).

The antiSMASH 5.0 program revealed 35 tentative BGCs of the secondary metabolites present in the S. rubrisoli Inha501

${ }^{1}$ http://igem.or.kr chromosome (Blin et al., 2019). BGC \# 22 was predicted to be the most likely BGC to synthesize the target linear polyene compound (Figure 4C). Bioinformatic analysis of BGC \#22 predicted the typical biosynthetic pathway of the target polyene compound and revealed a significant similarity to the BGC of the previously reported neotetrafibricin A (NTF A) (Table 2 and Supplementary Figure 3). LC-MS analysis of the purified target polyene compound ( $>90 \%$ purity) in the S. rubrisoli Inha501 culture showed a signal at $\mathrm{m} / \mathrm{z} 1226.73$ (calculated mass, 1227.73), which is the same as the signal at $\mathrm{m} / \mathrm{z} 1226.72$ for $\left[\mathrm{C}_{67} \mathrm{H}_{104} \mathrm{NO}_{19}\right]^{-}$of NTF A (Zhang et al., 2017; Supplementary Figure 4).

The PKS gene (Inha501-4694) of BGC \#22 was disrupted in the $S$. rubrisoli Inha501 chromosome to confirm that BGC \#22 is responsible for biosynthesis of the NTF A-like compound (Figure 5A and Supplementary Figure 5). As expected, the BGC \#22 PKS gene-disrupted mutant strain failed to produce NTF A-like compound, suggesting that the BGC \#22 is indeed responsible for the biosynthesis of NTF A-like compound in S. rubrisoli Inha501 (tentatively called Inha-neotetrafibricin A, I-NTF) (Yoo et al., 2011; Hong et al., 2013; Zhang et al., 2017; Figure 5B and Supplementary Figure 6).

\section{Biological Activities of I-NTF}

The purified I-NTF was evaluated for its antifungal activity by in vitro assays using a paper disc. The antifungal activity of I-NTF was confirmed in C. albicans and 11 phytopathogenic fungi. The size of the inhibition zone was larger than the controls, 


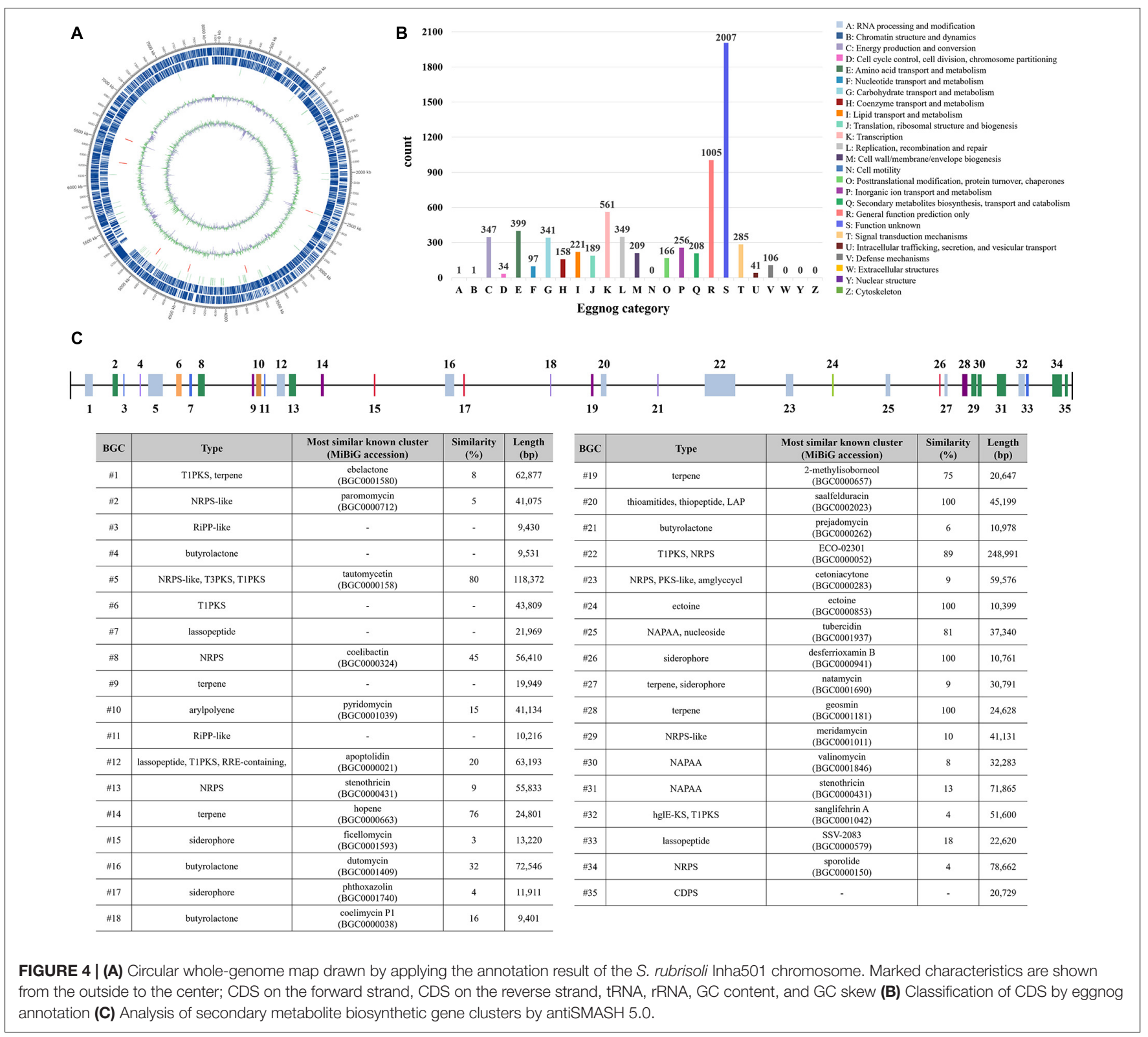

Amphotericin B and Nystatin A1 (Supplementary Figure 7). The antifungal activity was measured by examining the MIC (minimum inhibitory concentration) evaluation assays of the purified I-NTF using the colorimetric change in the RPMI-1640 media containing the fungus (Clinical and Laboratory Standards Institute, 2008; Supplementary Figure 8). The MIC of I-NTF against $C$. albicans, $A$. niger, and $F$. oxysporum was lower than nystatin A1 but higher than that of amphotericin B (Table 3). Interestingly, the MIC value of I-NTF against $F$. verticillioides, F. semitectum, C. lunata, and A. alternata was lower than nystatin A1 and amphotericin B (Table 3). In summary, the antifungal activity of I-NTF produced by $S$. rubrisoli Inha501 against 12 fungi (containing 11 phytopathogenic fungi) was confirmed.

\section{DISCUSSION}

In place of synthetic fungicides containing various environmental and health issues, considerable research for the isolation and application of eco-friendly microorganisms has been conducted. In this study, activity-based screening allowed the isolation of 149 strains of antifungal Streptomyces that exhibited antifungal activity out of the 2,419 strains tested. Existing activitybased screening strategies had limitations that the type and chemical structure of target compounds produced from selected strains were unknown until accurate structural analysis was completed. PCR screening approaches using the primers specific to polyene specific $\mathrm{P} 450$ genes were also implemented to select the novel strain producing a specific structural family, 
TABLE 2 | Annotation of the ORFs in the I-NTF gene cluster.

\begin{tabular}{|c|c|c|c|c|}
\hline ORF & Size $^{a}$ & Predicted function & S. aizunensis and S. neyagawaensis homolog ${ }^{b}$ & Accession number \\
\hline I-NTF A & 234 & Thioesterase & ORF1 (65/75\%) & AAX98176.1 \\
\hline I-NTF B & 920 & Transcriptional regulator & ORF2 (55/65\%) & AAX98177.1 \\
\hline I-NTF C & 210 & Response regulator & ORF3 (75/84\%) & AAX98178.1 \\
\hline I-NTF D & 417 & Sensor kinase & ORF4 (65/76\%) & AAX98179.1 \\
\hline I-NTF E & 182 & Putative membrane protein & ORF5 (64/75\%) & AAX98180.1 \\
\hline I-NTF F & 163 & Putative membrane protein & ORF6 (82/89\%) & AAX98181.1 \\
\hline I-NTF G & 520 & Putative membrane protein & ORF7 (63/75\%) & AAX98182.1 \\
\hline I-NTF H & 373 & Glycosyltransferase & ORF8 (74/82\%) & AAX98183.1 \\
\hline I-NTF PKS I & 9792 & Type I PKS & Tfb1 (72\%/80\%) & BAW35651.1 \\
\hline I-NTF PKS || & 9298 & Type I PKS & Tfb2 (68/76\%) & BAW35652.1 \\
\hline I-NTF PKS I|| & 6493 & Type I PKS & Tfb3 (72/79\%) & BAW35653.1 \\
\hline I-NTF PKS IV & 1633 & Type I PKS & Tfb4 (74/83\%) & BAW35654.1 \\
\hline I-NTF PKS V & 5121 & Type I PKS & Tfb5 (74/81\%) & BAW35655.1 \\
\hline I-NTF PKS VI & 5309 & Type I PKS & Tfb6 (71/79\%) & BAW35656.1 \\
\hline I-NTF PKS VII & 3169 & Type I PKS & Tfb7 (78/85\%) & BAW35657.1 \\
\hline I-NTF PKS VIII & 7352 & Type I PKS & Tfb8 (75/82\%) & BAW35658.1 \\
\hline I-NTF PKS IX & 3755 & Type I PKS & Tfb9 (74/81\%) & BAW35659.1 \\
\hline |-NTF | & 317 & Acyltransferase & ORF18 (66/75\%) & AAX98193.1 \\
\hline I-NTF J & 298 & Putative membrane protein & ORF19 (65/76\%) & AAX98194.1 \\
\hline I-NTF K & 349 & ABC transporter & ORF20 (68/79\%) & AAX98195.1 \\
\hline I-NTF L & 335 & Sugar dehydratase/epimerase & ORF21 (58/67\%) & AAX98196.1 \\
\hline I-NTFM & 210 & Sugar epimerase & ORF22 (69/82\%) & AAX98197.1 \\
\hline I-NTF N & 355 & Sugar nucleotidyltransferase & ORF23 (74/81\%) & AAX98198.1 \\
\hline I-NTF O & 328 & Sugar dehydratase/epimerase & ORF24 (77/82\%) & AAX98199.1 \\
\hline I-NTF P & 227 & Thioesterase & ORF25 (55/66\%) & AAX98200.1 \\
\hline I-NTF Q & 467 & Acyl CoA ligase & ORF26 (78/87\%) & AAX98201.1 \\
\hline I-NTF R & 544 & Amine oxidase & ORF27 (76/83\%) & AAX98202.1 \\
\hline I-NTF S & 226 & Phosphopantetheinyl transferase & ORF28 (56/67\%) & AAX98203.1 \\
\hline I-NTF T & 303 & Metallophosphoesterase & ORF29 (80/84\%) & AAX98204.1 \\
\hline I-NTF U & 952 & Transcriptional regulator & ORF30 (51/64\%) & AAX98205.1 \\
\hline I-NTF V & 533 & Carboxylase/carboxyltransferase & ORF31 (86/91\%) & AAX98206.1 \\
\hline I-NTF W & 332 & Amidinohydrolase & ORF32 (79/90\%) & AAX98207.1 \\
\hline
\end{tabular}

a Size in the number of amino acids.

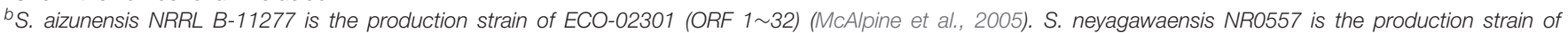
neotetrafibricin A (Tfb 1 9) (Zhang et al., 2017). The homolog shows amino acid sequence identity\%/similarity\%.

such as polyene compounds (Hwang et al., 2007). Complete genome sequencing of the selected strain complemented the weakness of the activity-based strategy, and the BGC analysis made it feasible to predict the tentative structure of the target compound.

Through the whole genome sequence analysis of $S$. rubrisoli Inha501, 35 putative BGCs were predicted, of which seven BGCs contained the PKS genes as the core biosynthetic genes. Thanks to the co-linearity nature of the sequence, the biosynthetic pathway of the type I PKS system and its structure are relatively easy to predict in accordance with the usual rules of the PKS multidomain modular system. Through anti-SMASH analysis of all BGCs present in the S. rubrisoli Inha501 strains, it was predicted that the target antifungal compound encoded by the BGC \#22 was the previously reported giant linear polyene compound called NTF A (Zhang et al., 2017). The compound produced by S. rubrisoli Inha501 was tentatively named I-NTF (Inha neotetrafibricin) until a more definite structure analysis was completed. The gene-knockout experiments confirmed that BGC \#22 is responsible for the biosynthesis of an I-NTF. Interestingly, I-NTF biosynthetic gene knockout showed a decrease in overall antifungal activity in S. rubrisoli Inha501, but the activity did not disappear completely, suggesting that there might be additional functional antifungal BGCs in S. rubrisoli Inha501, such as BGC \#5 (80\% similarity to tautomycetin) and BGC \#25 (81\% similarity to tubercidin) (Choi et al., 2017; Liu et al., 2018).

The newly isolated $S$. rubrisoli Inha501 confirmed its antifungal activity against various phytopathogenic fungal strains and showed its potential applications as a bio-control agent through pot-test and field-test for actual crops, such as strawberries, tomatoes, and red peppers. Optimization of the culture condition and its formulation study is currently being pursued for the development of microbial fungicides of S. rubrisoli Inha501. The synergistic approach between 


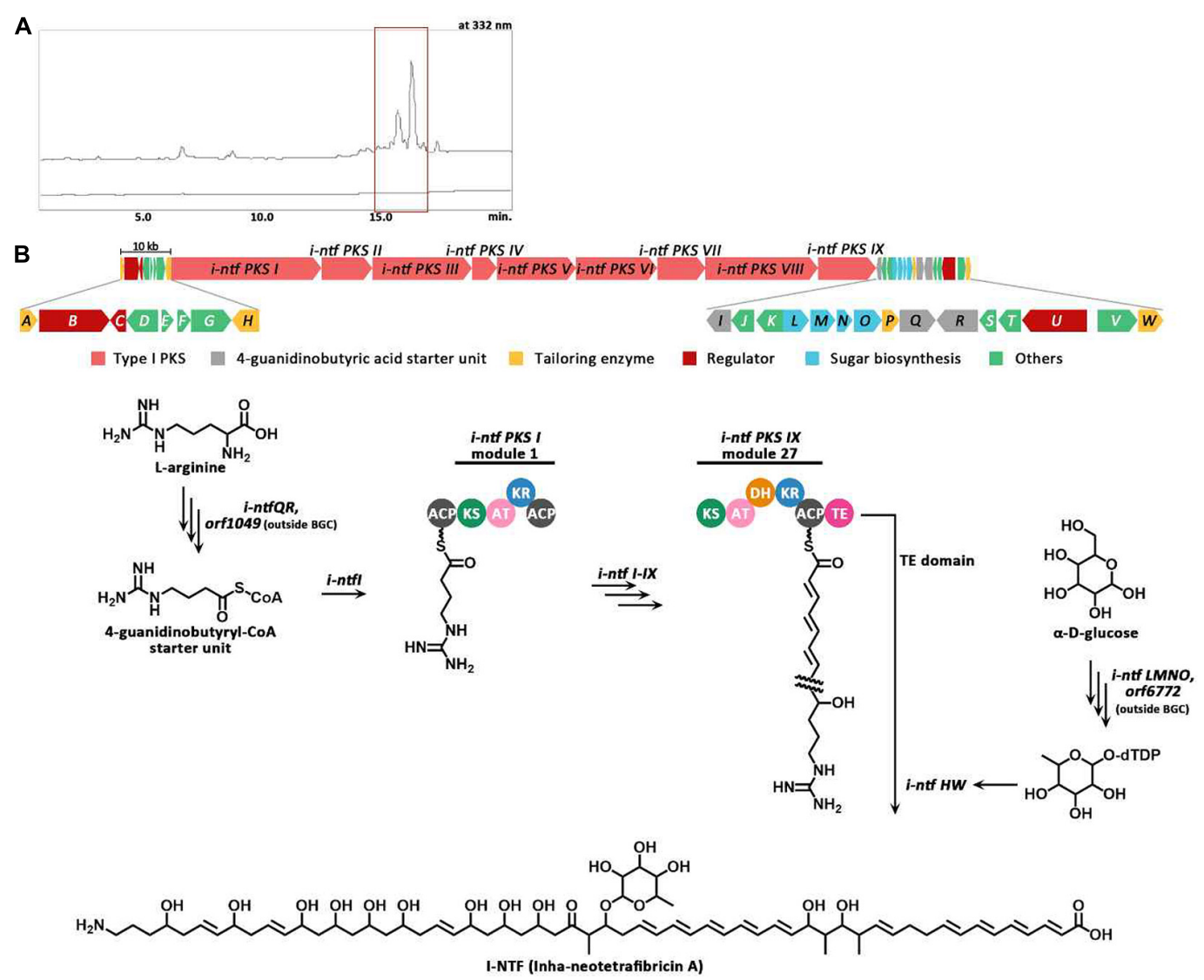

FIGURE 5 | (A) HPLC chromatograms of the cultures from the wild-type S. rubrisoli Inha501 (upper line) and the $i$-ntf PKS I disrupted S. rubrisoli Inha501 mutant (Iower line). The asterisked peak is confirmed to be the I-NTF and the small peak in front of I-NTF peak is believed to the derivative or degradation product of I-NTF (B) ORFs encoded in the putative I-NTF biosynthetic gene cluster and proposed biosynthetic pathway to I-NTF (more details of ORFs are listed in Table 2).

TABLE 3 | In vitro antifungal activity.

Antifungal activity (MIC $\left.{ }^{a}, \mu \mathrm{g} / \mathrm{ml}\right)$

\begin{tabular}{lccc}
\hline Strain & Amphotericin B & Nystatin A1 & I-NTF \\
\hline C. albicans ATCC 14053 & 0.5 & 4 & 2 \\
A. niger ATCC 9642 & 0.5 & 4 & 2 \\
F. oxysporum KACC 40051 & 2 & 8 & 2 \\
F. oxysporum KACC 42795 & 1 & 8 & 4 \\
F. verticillioides KCTC 6065 & 1 & 8 & $<0.25$ \\
F. semitetum KCTC 16672 & 1 & 4 & 0.5 \\
A. alternata KACC 40019 & $>16$ & $>16$ & 4 \\
C. lunata KACC 40861 & 16 & $>16$ & 4 \\
C. gloeosporioides KACC 40003 & 0.5 & 2 & 4 \\
B. cinerea KACC 40574 & 1 & 2 & 16
\end{tabular}

${ }^{a} \mathrm{MIC}$, minimum inhibitory concentration (values resulting in no visible growth of the fungus).

activity-based screening and genome analysis highlights the efficient isolation of novel strains, such as S. rubrisoli Inha501, which contains a giant linear polyene BGC and a strong antiphytopathogenic compound to protect various crops growing in the soil environments.

\section{DATA AVAILABILITY STATEMENT}

The datasets presented in this article are not readily available because the government-funded whole genome sequence is deposited in the local members-only genome database. Requests to access the datasets should be directed to eungsoo@inha.ac.kr.

\section{AUTHOR CONTRIBUTIONS}

H-SP, S-SC, and E-SK designed the experiments. H-SP, H-JN, and S-HK performed the experiments. H-SP and E-SK wrote the manuscript. All authors contributed to the article and approved the submitted version.

\section{FUNDING}

This study was supported by Agricultural Microbiome R\&D Program, Ministry of Agriculture, Food and Rural Affairs, Republic of Korea (as part of the (multi-ministerial) Genome Technology to Business Translation Program No. 91800804). This work was also funded by the National Research 
Foundation of Korea (NRF-2021R1A2C2012203). The soil actinomycetes strains were kindly provided by the grant (NRF-2013M3A9A5076601) from a study on the strategies of improving the value of microbial resources funded by the Ministry of Science and ICT of the Korea Government.

\section{REFERENCES}

Ab Rahman, S. F. S., Singh, E., Pieterse, C. M., and Schenk, P. M. (2018). Emerging microbial biocontrol strategies for plant pathogens. Plant Sci. 267, 102-111. doi: $10.1016 /$ j.plantsci.2017.11.012

Abbasi, S., Safaie, N., Sadeghi, A., and Shamsbakhsh, M. (2019). Streptomyces strains induce resistance to Fusarium oxysporum f. sp. lycopersici race 3 in tomato through different molecular mechanisms. Front. Microbiol. 10:1505. doi: 10.3389/fmicb.2019.01505

Awakawa, T., Fujioka, T., Zhang, L., Hoshino, S., Hu, Z., Hashimoto, J., et al. (2018). Reprogramming of the antimycin NRPS-PKS assembly lines inspired by gene evolution. Nat. Commun. 9, 1-10. doi: 10.1038/s41467-018-05 $877-\mathrm{z}$

Blin, K., Shaw, S., Steinke, K., Villebro, R., Ziemert, N., Lee, S. Y., et al. (2019). antiSMASH 5.0: updates to the secondary metabolite genome mining pipeline. Nucleic Acids Res. 47, W81-W87. doi: 10.1093/nar/gkz310

Bu, Q. T., Yu, P., Wang, J., Li, Z. Y., Chen, X. A., Mao, X. M., et al. (2019). Rational construction of genome-reduced and high-efficient industrial Streptomyces chassis based on multiple comparative genomic approaches. Microb. Cell Fact. 18, 1-17. doi: 10.1186/s12934-019-1055-7

Caffrey, P., De Poire, E., Sheehan, J., and Sweeney, P. (2016). Polyene macrolide biosynthesis in streptomycetes and related bacteria: recent advances from genome sequencing and experimental studies. Appl. Microbiol. Biotechnol. 100, 3893-3908. doi: 10.1007/s00253-016-7474-z

Choi, S. S., Nah, H. J., Pyeon, H. R., and Kim, E. S. (2017). Biosynthesis, regulation, and engineering of a linear polyketide tautomycetin: a novel immunosuppressant in Streptomyces sp. CK4412. J. Ind. Microbiol. Biotechnol. 44, 555-561. doi: 10.1007/s10295-016-1847-2

Clinical and Laboratory Standards Institute (2008). Reference for Broth Dilution Antifungal Susceptibility Testing of Yeasts; Approved Standard, 3rd Edn. Wayne, PA: Clinical and Laboratory Standards Institute document, M27-M23.

Dean, R., Van Kan, J. A., Pretorius, Z. A., Hammond-Kosack, K. E., Di Pietro, A., Spanu, P. D., et al. (2012). The Top 10 fungal pathogens in molecular plant pathology. Mol. Plant Pathol. 13, 414-430. doi: 10.1111/j.1364-3703.2011. 00783.x

Guo, J., Schlingmann, G., Carter, G. T., Nakanishi, K., and Berova, N. (2000). Absolute stereochemistry of the strevertenes. Chirality 12, 43-51. doi: 10.1002/ (SICI) 1520-636X200012:1<43::AID-CHIR8<3.0.CO;2-N

Hong, H., Fill, T., and Leadlay, P. F. (2013). A common origin for guanidinobutanoate starter units in antifungal natural products. Angew. Chem. Int. Ed. Engl. 52, 13096-13099. doi: 10.1002/anie.201308136

Huerta-Cepas, J., Szklarczyk, D., Heller, D., Hernández-Plaza, A., Forslund, S. K., Cook, H., et al. (2019). eggNOG 5.0: a hierarchical, functionally and phylogenetically annotated orthology resource based on 5090 organisms and 2502 viruses. Nucleic Acids Res. 47, D309-D314. doi: 10.1093/nar/gky1085

Hwang, Y. B., Lee, M. Y., Park, H. J., Han, K., and Kim, E. S. (2007). Isolation of putative polyene-producing actinomycetes strains via PCR-based genome screening for polyene-specific hydroxylase genes. Process Biochem. 42, 102-107. doi: $10.1016 /$ j.procbio.2006.06.031

Jakubiec-Krzesniak, K., Rajnisz-Mateusiak, A., Guspiel, A., Ziemska, J., and Solecka, J. (2018). Secondary metabolites of actinomycetes and their antibacterial, antifungal, and antiviral properties. Pol. J. Microbiol. 67, 259-272. doi: 10.21307/pjm-2018-048

Kim, D. R., Cho, G., Jeon, C. W., Weller, D. M., Thomashow, L. S., Paulitz, T. C., et al. (2019). A mutualistic interaction between Streptomyces bacteria, strawberry plants, and pollinating bees. Nat. Commun. 10:4802. doi: 10.1038/ s41467-019-12785-3

Kramer, C. Y. (1957). Extension of multiple range tests to group correlated adjusted means. Biometrics 13, 13-18.

\section{SUPPLEMENTARY MATERIAL}

The Supplementary Material for this article can be found online at: https://www.frontiersin.org/articles/10.3389/fbioe. 2021.692340/full\#supplementary-material

Kumar, S., Stecher, G., and Tamura, K. (2016). MEGA7: molecular evolutionary genetics analysis version 7.0 for bigger datasets. Mol. Biol. Evol. 33, 1870-1874. doi: 10.1093/molbev/msw054

Lee, N., Hwang, S., Kim, J., Cho, S., Palsson, B., and Cho, B. K. (2020). Mini review: genome mining approaches for the identification of secondary metabolite biosynthetic gene clusters in Streptomyces. Comput. Struct. Biotechnol. J. 18, 1548-1556. doi: 10.1016/j.csbj.2020.06.024

Liu, Y., Gong, R., Liu, X., Zhang, P., Zhang, Q., Cai, Y. S., et al. (2018). Discovery and characterization of the tubercidin biosynthetic pathway from Streptomyces tubercidicus NBRC 13090. Microb. Cell Fact. 17:131. doi: 10.1186/s12934-0180978-8

Manivasagan, P., Venkatesan, J., Sivakumar, K., and Kim, S. K. (2014). Pharmaceutically active secondary metabolites of marine actinobacteria. Microbiol. Res. 169, 262-278. doi: 10.1016/j.micres.2013.07.014

McAlpine, J. B., Bachmann, B. O., Piraee, M., Tremblay, S., Alarco, A. M., Zazopoulos, E., et al. (2005). Microbial genomics as a guide to drug discovery and structural elucidation: ECO-02301, a novel antifungal agent, as an example. J. Nat. Prod. 68, 493-496. doi: 10.1021/np0401664

Neumann, A., Wieczor, M., Zielinska, J., Baginski, M., and Czub, J. (2016). Membrane sterols modulate the binding mode of amphotericin B without affecting its affinity for a lipid bilayer. Langmuir 32, 3452-3461. doi: 10.1021/ acs.langmuir.5b04433

Parnell, J. J., Berka, R., Young, H. A., Sturino, J. M., Kang, Y., Barnhart, D. M., et al. (2016). From the lab to the farm: an industrial perspective of plant beneficial microorganisms. Front. Plant. Sci. 7:1110. doi: 10.3389/fpls.2016.01110

Rural Development Administration (2008). Pesticide Registration Test Standards and Methods. Available online at: https://www.law.go.kr/LSW/admRulInfoP. do?admRulSeq=2000000060683 (accessed Febuary 11, 2008).

Scott, R. A., and Milliken, G. A. (1993). A SAS program for analyzing augmented randomized complete-block designs. Crop Sci. 33, 865-867.

Shi, L., Nwet, T. T., Ge, B., Zhao, W., Liu, B., Cui, H., et al. (2018). Antifungal and plant growth-promoting activities of Streptomyces roseoflavus strain NKZ-259. Biol. Control 125, 57-64. doi: 10.1016/j.biocontrol.2018.06.012

Yoo, H. G., Kwon, S. Y., Karki, S., and Kwon, H. J. (2011). A new route to dTDP6-deoxy-L-talose and dTDP-L-rhamnose: dTDP-L-rhamnose 4-epimerase in Burkholderia thailandensis. Bioorg. Med. Chem. Lett. 21, 3914-3917. doi: 10. 1016/j.bmcl.2011.05.030

Zerbe, G. O. (1979). Randomization analysis of the completely randomized design extended to growth and response curves. J. Am. Stat. Assoc. 74, 215-221.

Zerikly, M., and Challis, G. L. (2009). Strategies for the discovery of new natural products by genome mining. ChemBioChem 10, 625-633. doi: 10.1002/cbic. 200800389

Zhang, L., Hashimoto, T., Qin, B., Hashimoto, J., Kozone, I., Kawahara, T., et al. (2017). Characterization of giant modular PKSs provides insight into genetic mechanism for structural diversification of aminopolyol polyketides. Angew. Chem. Int. Ed. Engl. 56, 1740-1745. doi: 10.1002/anie.201611371

Ziemert, N., Alanjary, M., and Weber, T. (2016). The evolution of genome mining in microbes-a review. Nat. Prod. Rep. 33, 988-1005. doi: 10.1039/c6np00025h

Conflict of Interest: The authors declare that the research was conducted in the absence of any commercial or financial relationships that could be construed as a potential conflict of interest.

Copyright (c) 2021 Park, Nah, Kang, Choi and Kim. This is an open-access article distributed under the terms of the Creative Commons Attribution License (CC BY). The use, distribution or reproduction in other forums is permitted, provided the original author(s) and the copyright owner(s) are credited and that the original publication in this journal is cited, in accordance with accepted academic practice. No use, distribution or reproduction is permitted which does not comply with these terms. 